\title{
Resistive Switching Properties of Samarium Oxide Resistive Random Access Memories
}

\author{
Chien-Min Cheng, ${ }^{1}$ Kai-Huang Chen, ${ }^{2}$ Fuh-Cheng Jong, ${ }^{1}$ Mei-Li Chen, ${ }^{3 *}$ \\ Hsuan-Hung Li, ${ }^{3}$ Han-Chin Chen, ${ }^{3}$ and Po-Yu Hsieh ${ }^{3}$ \\ ${ }^{1}$ Department of Electronic Engineering, Southern Taiwan University of Science and Technology, \\ No. 1, Nan-Tai Street, Yungkang Dist., Tainan City 710, Taiwan, R.O.C. \\ ${ }^{2}$ Department of Digital Game and Animation Design, Tung Fang Design University, \\ No. 110, Dongfang Rd., Hunei Dist., Kaohsiung City 82941, Taiwan, R.O.C. \\ ${ }^{3}$ Department of Electro-Optical Engineering, Southern Taiwan University of Science, \\ No. 1, Nan-Tai Street, Yungkang Dist., Tainan City 710, Taiwan, R.O.C.
}

(Received August 1, 2017; accepted November 2, 2017)

Keywords: RF magnetron sputtering method, resistive random access memory, samarium oxide

The bipolar switching and electrical conduction properties of the samarium oxide resistive random access memory (RRAM) device were investigated in this study. By the RF magnetron sputtering method (Sm target, RF power $75 \mathrm{~W}$, chamber pressure $20 \mathrm{mTorr}$, and different oxygen concentrations), samarium oxide thin films were deposited on an indium tin oxide (ITO)/glass substrate to form a $\mathrm{SmO}_{X} / \mathrm{ITO}$ structure. Using the metal mask and E-beam evaporation, the aluminum upper electrode was then deposited on it to form an $\mathrm{Al} / \mathrm{SmO}_{X} / \mathrm{ITO}$ metal/insulation/ metal (MIM) structure. Additionally, conventional thermal annealing treatments were used to improve grain growth and the properties of the thin films. From the results, greater than $10^{2}$ on/off ratio and bipolar switching cycling behaviors of two stable states were obtained. As the operating voltage is low, the conduction mechanisms for low- and high-resistance states are dominated by ohmic conduction behavior for both SET and RESET states. However, as a higher operating voltage is applied, parts of conduction mechanisms will change to space charge limit conduction behaviors. The proposed $\mathrm{Al} / \mathrm{SmO}_{X} / \mathrm{ITO} \mathrm{RRAM}$ devices also exhibit good memory window and stable bipolar switching properties during 100 cycles whereas the RF power is 75 $\mathrm{W}$ and the oxygen concentration is $10 \mathrm{sccm}$.

\section{Introduction}

As the flash memory and dynamic random access memory (DRAM) are scaled down, the memories reveal charge loss and reach their limiting physical dimensions gradually. ${ }^{(1,2)}$ Recently, resistive random access memory (RRAM) devices have been shown to possess advantages such as nonvolatility, high operation speed, multiple-state possibility, small bit size, and low power consumption. ${ }^{(3,4)}$ Hence, RRAMs have been identified as a prospective candidate for replacing traditional memory devices, and have been widely used in portable

*Corresponding author: e-mail: cmli@stust.edu.tw

http://dx.doi.org/10.18494/SAM.2018.1766 
electronic systems, such as cellular phones, personal digital assistants (PDAs), flash storage, and digital cameras.

The basic structure of RRAMs is a dielectric layer clamped by top and bottom electrodes [metal/insulation/metal (MIM) structure]. The memory effect of RRAMs is fulfilled through the switching characteristics between a high-resistance (OFF) state (HRS) and a low-resistance (ON) state (LRS) of the dielectric layer. In the past, various materials were discovered for this dielectric layer, such as chalcogenide, ${ }^{(5-7)}$ oxide-based, ${ }^{(8-10)}$ carbide, ${ }^{(1)}$ and amorphous silicon. ${ }^{(12)}$ However, except for the above RRAM materials, samarium oxide $(\mathrm{SmO})$ is another important rare-earth material owing to its high thermal stability and large band gaps. There is still insufficient information for discussing the resistive switching properties of $\mathrm{SmO}_{X} \mathrm{RRAMs}$ to date. Hence, in this research, we investigate the resistive switching properties, bipolar switching properties, current transport mechanisms, and the on/off ratio of $\mathrm{Al} / \mathrm{SmO}_{X} /$ indium tin oxide (ITO) RRAMs for future memory applications.

\section{Experimental Procedures}

With a 2 inch ITO target and different oxygen $\left(\mathrm{O}_{2}\right) /$ Argon $(\mathrm{Ar})$ concentrations $\left(\mathrm{O}_{2} / \mathrm{Ar}=\right.$ $0 \mathrm{sccm} / 10 \mathrm{sccm}, 5 \mathrm{sccm} / 10 \mathrm{sccm}, 10 \mathrm{sccm} / 10 \mathrm{sccm}$, and $15 \mathrm{sccm} / 10 \mathrm{sccm}$ ), the $\mathrm{SmO}_{X}$ thin films were deposited onto the ITO/glass substrate by the RF magnetron sputtering method and with optimal deposition parameters of RF power ( $75 \mathrm{~W})$, chamber pressure (20 mTorr), and sputtering time (10 min). Using SHIMADZU XRD-6000, the X-ray diffraction (XRD) patterns of the $\mathrm{SmO}_{X}$ thin films were recorded to determine their crystalline quality for the $2 \theta$-degree range of $20-60^{\circ}$, and their surface morphologies were observed by field emission scanning electron microscopy (FE-SEM).

To complete the MIM (Al/SmO $/$ /ITO) structure, an array of aluminum (Al) circular top electrodes (thickness is $300 \mathrm{~nm}$ and diameter is $0.1 \mathrm{~cm}$ ) was formed by E-beam evaporation in a vacuum system $\left(5 \times 10^{-6}\right.$ Torr). The proposed $\mathrm{Al} / \mathrm{SmO}_{X} / \mathrm{ITO} \mathrm{RRAM}$ devices are schematically shown in Fig. 1. The current versus applied voltage $(I-V)$ characteristics of the $\mathrm{Al} / \mathrm{SmO}_{X} /$ ITO RRAM were measured using a probe station and a semiconductor parameter analyzer (HP4156C). Furthermore, the number of switching cycles was measured at room temperature, and the current conduction mechanisms of SET and RESET regions were also analyzed and investigated.

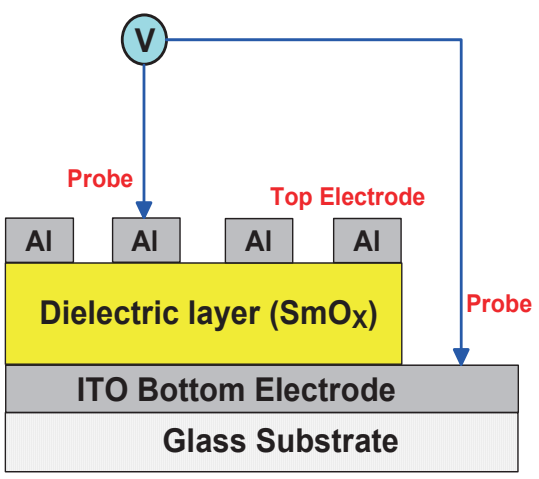

Fig. 1. (Color online) Simulated antenna gains for different distances between metal reflector and proposed antenna. 


\section{Results and Discussion}

Figure 2 shows the XRD patterns of the as-deposited $\mathrm{SmO}_{X}$ thin films for RF power of $75 \mathrm{~W}$ prepared at different oxygen concentrations. From the XRD patterns, we found that all the $\mathrm{SmO}_{X}$ thin films exhibited a clear peak at about $35^{\circ}$, corresponding to the (400) textured orientation. ${ }^{(13-15)}$ This means that completed ITO phases had been obtained according to the optimal sputtering parameters. Because argon is used to generate plasma during the sputtering process, the $\mathrm{Ar} / \mathrm{O}_{2}$ ratio thus can also be used to adjust the thickness of $\mathrm{SmO}_{X}$ thin films.

Figure 3 shows the FE-SEM surface morphologies of $\mathrm{SmO}_{X}$ thin films for different oxygen concentrations. It can be seen from Fig. 3(a) that nonuniform grains and pores can be found for a $0 \mathrm{sccm}$ specimen. As shown in Figs. 3(b) and 3(c), the surfaces become dense as the oxygen

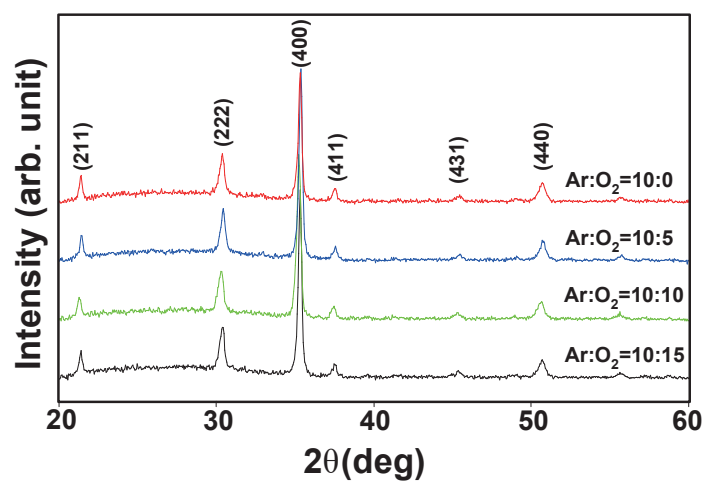

Fig. 2. (Color online) XRD patterns of the as-deposited $\mathrm{SmO}_{X}$ thin films for different oxygen concentrations.
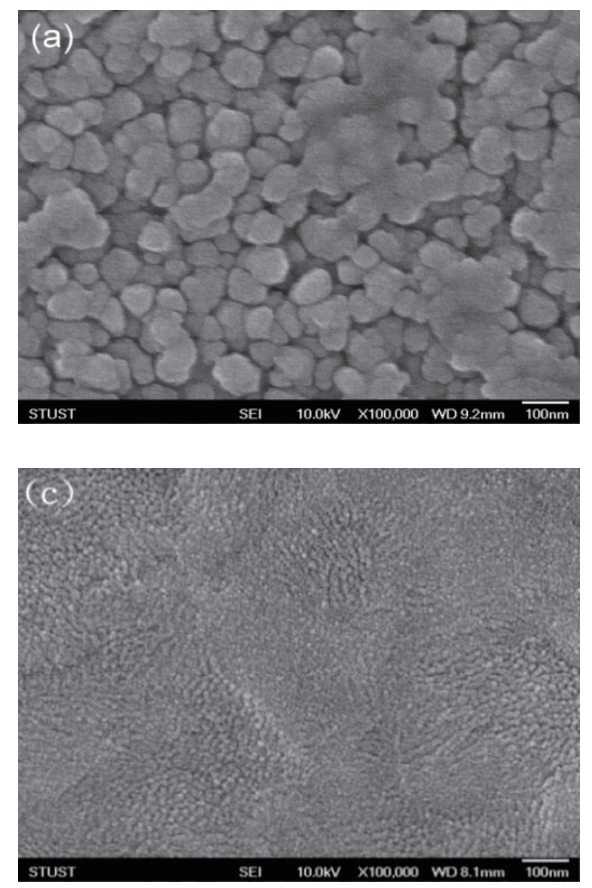
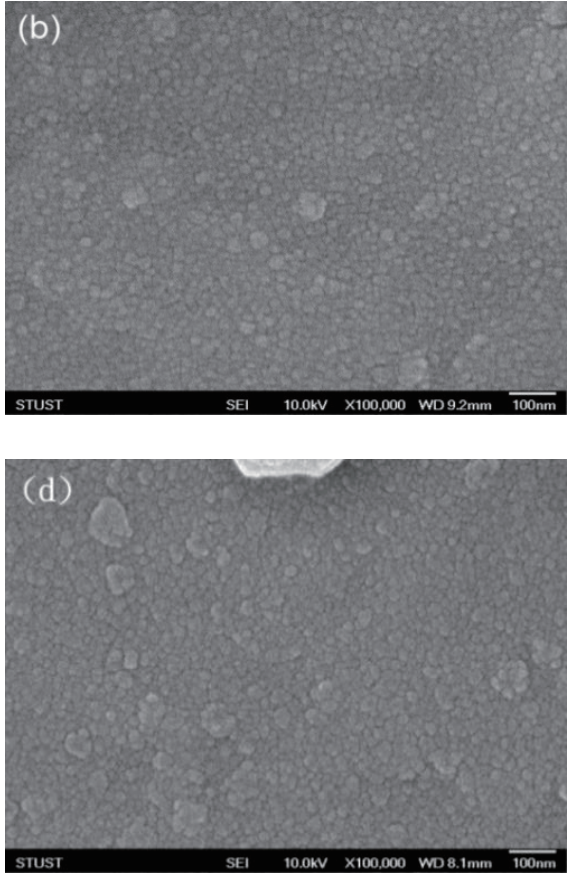

Fig. 3. Surface morphologies of $\mathrm{SmO}_{X}$ thin films for different oxygen concentrations. (a) 0, (b) 5, (c) 10, and (d) 15 sccm. 
concentration increased to 5 and $10 \mathrm{sccm}$. However, as the oxygen concentration increased to $15 \mathrm{sccm}$, the grains become more nonuniform. Additionally, the oxygen vacancies are the dominant factor for the current transmission paths and were generated during the sputtering process. The oxygen reacts with Sm target materials and parts of the oxygen might fill oxygen vacancies, which will break the current transmission paths and also cause the variation of the $X$ value in $\mathrm{SmO}_{X}$. Hence, too much oxygen will fill oxygen vacancies and cause the decrease in the number of oxygen vacancies, which will affect the number of current transmission paths and also the insulating properties of the $\mathrm{SmO}_{X}$ layer, probably suggesting that the optimal oxygen concentration is $10 \mathrm{sccm}$. The cross-sectional image of the proposed RRAM is shown in Fig. 4. Clearly, the thicknesses of the $\mathrm{Al}$ top electrode and $\mathrm{SmO}_{X}$ layer are about 179 and $35 \mathrm{~nm}$, respectively.

During the RF sputtering process, defects and oxygen vacancies of as-deposited $\mathrm{SmO}_{X}$ thin films were filled and compensated by oxygen. For the $I-V$ characteristics shown in Fig. 5, by sweeping the bias to negative over the set voltage, a gradual increase in current was presented to switch the cells from the HRS to the LRS (set process). Additionally, the cell turns back to HRS on applying a larger positive bias than the reset voltage (reset process). Moreover, it can be found that all the RRAMs exhibit clear bipolar behaviors, and all the on/off ratios of both SET and RESET states are greater than 102. Furthermore, it exhibited less than $3 \mathrm{~V}$ operating voltage and good memory switching properties.

It was reported that oxygen contents play an important role in the resistive switching of RRAM devices. ${ }^{(16-18)}$ In the filament model, the formation and rupture of the conducting filaments are the main switching mechanisms. Since the switching properties of the $\mathrm{SmO}_{X}$ film are highly correlated with the number of oxygen ions and vacancies within the electrode and thin film, the oxygen concentration will play an important role in the resistive switching phenomenon of this $\mathrm{SmO}_{X}$ RRAM device.

For RRAMs, many models had been suggested to illustrate their conductive mechanisms in the past, such as the modification of Schottky barrier height by trapped charge carriers, ${ }^{(19)}$ constitution of a conductive filamentary path, ${ }^{(20)}$ migration of oxygen vacancies, ${ }^{(21)}$ and carriers tunneling between crystalline defects. ${ }^{(22)}$ Furthermore, many more researchers have attempted to optimize the resistive switching properties of RRAMs. The two most popular

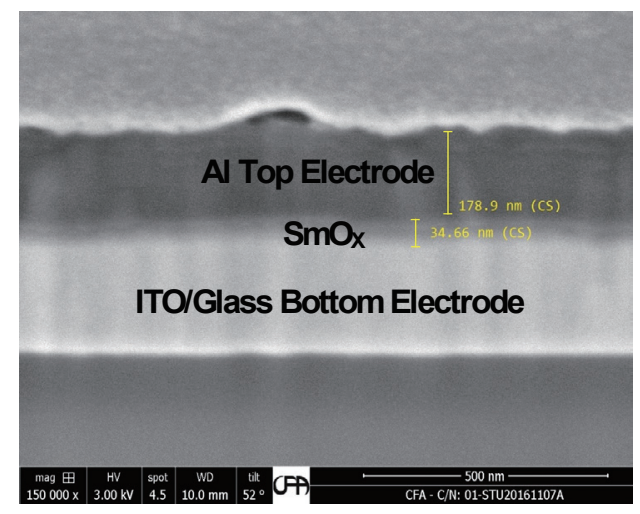

Fig. 4. (Color online) Cross-sectional image of $\mathrm{SmO}_{X}$ thin films ( $\mathrm{RF}$ power $\left.=75 \mathrm{~W}, \mathrm{O}_{2}=10 \mathrm{sccm}\right)$. 


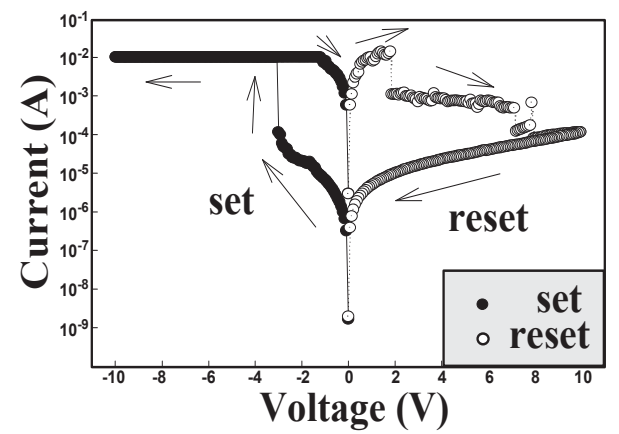

(a)

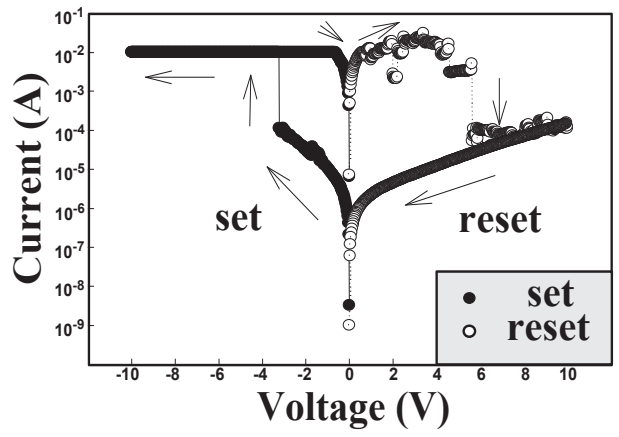

(c)

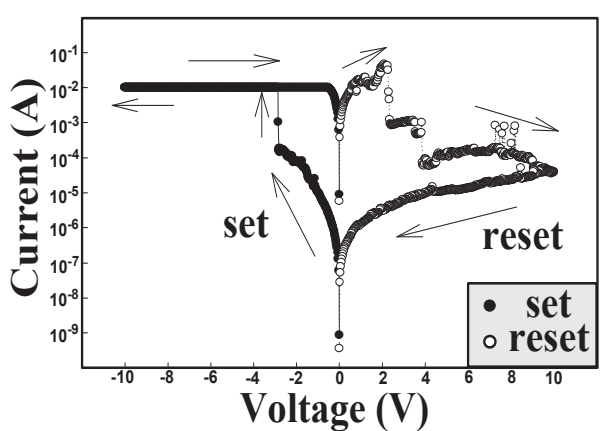

(b)

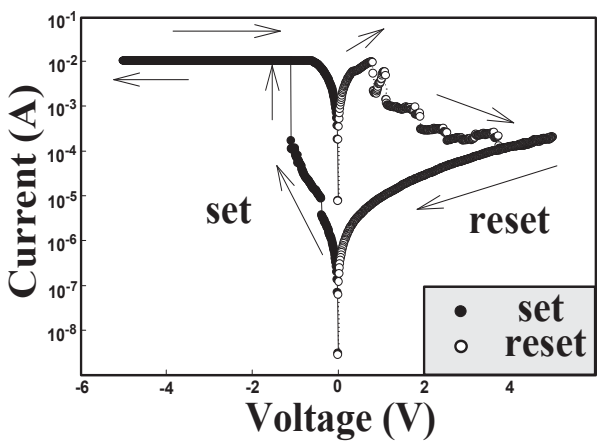

(d)

Fig. 5. $I-V$ characteristics of $\mathrm{Al} / \mathrm{SmO}_{X} / \mathrm{ITO} \mathrm{RRAM}$ for different oxygen concentrations $(\mathrm{RF}$ power $=75 \mathrm{~W})$. (a) 0 , (b) 5 , (c) 10 , and (d) $15 \mathrm{sccm}$.

conductive mechanisms of RRAMs are the ohmic conduction [Eq. (1)] and the space charge limit conduction (SCLC) behaviors [Eq. (2)]. ${ }^{(23)}$ As the RF power was 75 W, Fig. 6 shows the current-voltage characteristics of $0,5,10$, and $15 \mathrm{sccm} \mathrm{O} \mathrm{O}_{2} \mathrm{Al} / \mathrm{SmO}_{X} / \mathrm{ITO} \mathrm{RRAMs}$ in terms of $\operatorname{Ln}(I)$ and $\operatorname{Ln}(V)$. We can observe that as the operating voltages were low, the current conduction mechanism was ohmic conduction behavior (external electric field dominated, slope $\approx 1$ ) for both SET and RESET states. However, for higher operating voltages, decreasing current can be observed owing to parts of defects filled by the carriers. Thus, parts of conduction mechanisms will change to SCLC behavior (slope $\approx 2$ ). However, for intermediate operating voltage $(1<$ slope $<2)$, the conduction mechanisms were composed of both ohmic conduction as well as SCLC.

$$
\begin{gathered}
J=n q \mu E \exp \left(\frac{-\Delta E_{a c}}{k T}\right), \\
J=\frac{9 \varepsilon_{i} \mu V^{2}}{8 d^{3}} .
\end{gathered}
$$

Here, $J$ is current density, $n$ is electron concentration, $\mu$ is mobility of electrons, $E$ is applied electric field, $\Delta E_{a c}$ is the activation energy of electrons, $k$ is Boltzmann's constant, $\varepsilon_{i}$ is insulator dynamic permittivity, $d$ is the thickness of the insulator layer, and $V$ is applied voltage. 

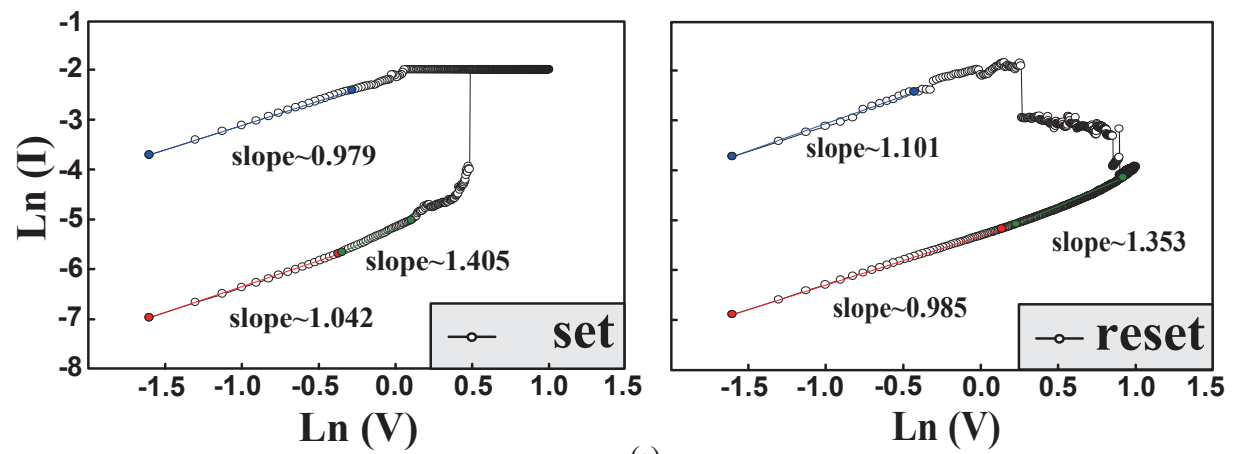

(a)
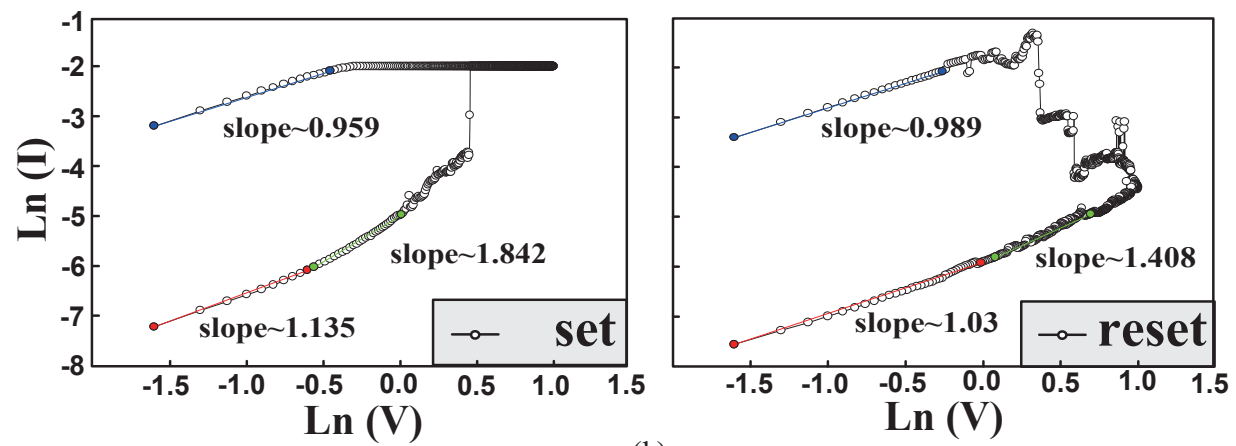

(b)
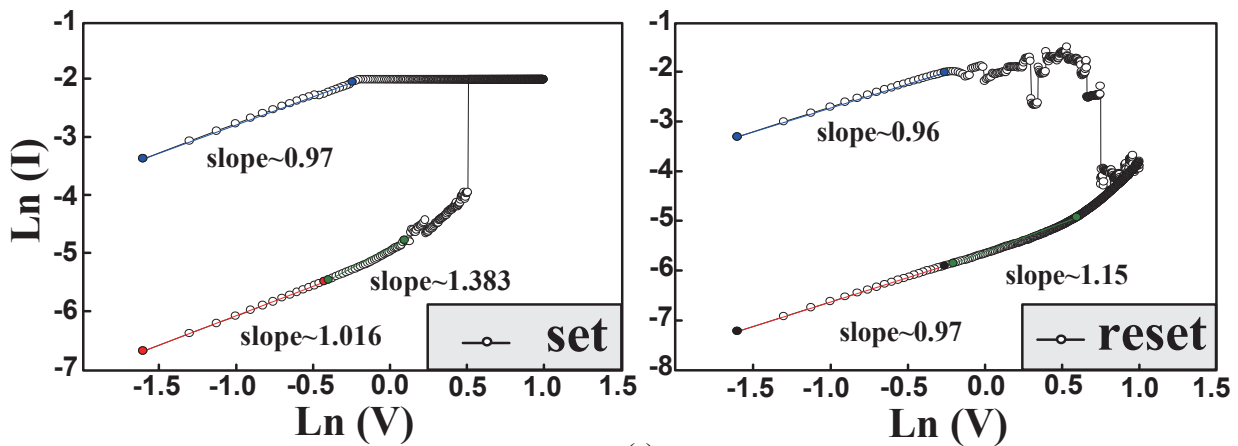

(c)
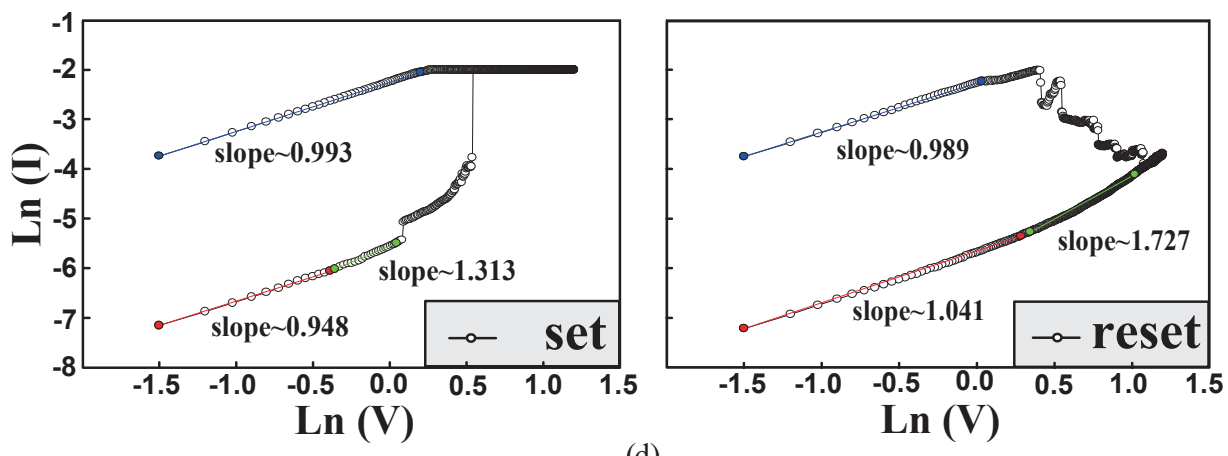

(d)

Fig. 6. (Color online) $\mathrm{Ln}(I)-\mathrm{Ln}(V)$ plots of $\mathrm{Al} / \mathrm{SmO}_{X} / \mathrm{ITO} \mathrm{RRAM}$ for different oxygen concentrations (RF power $=75 \mathrm{~W}$ ). (a) 0 , (b) 5 , (c) 10 , and (d) $15 \mathrm{sccm}$. 


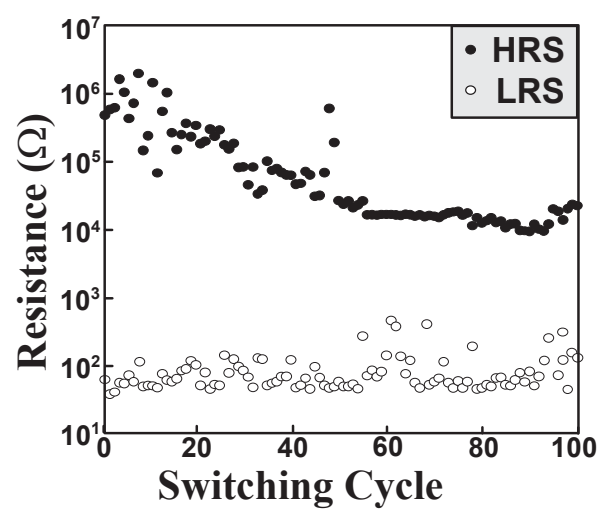

Fig. 7. Switching cycle of the $\mathrm{Al} / \mathrm{SmO}_{X} / \mathrm{ITO} \mathrm{RRAM}\left(\mathrm{RF}\right.$ power $\left.=75 \mathrm{~W}, \mathrm{O}_{2}=10 \mathrm{sccm}\right)$.

As the RF power is $75 \mathrm{~W}$ and the oxygen concentration is $10 \mathrm{sccm}$, the switching endurance characteristics of the $\mathrm{Al} / \mathrm{SmO}_{X} / \mathrm{ITO} \mathrm{RRAM}$ were measured and shown in Fig. 7. There was a slight fluctuation of resistance in the HRS (resistance is about $10^{4}-10^{6} \Omega$ ) and LRS (resistance is about $10^{2} \Omega$ ) states. Thus, the device reveals stable bipolar switching properties and greater than $10^{2} \mathrm{HRS} / \mathrm{LRS}$ resistance ratio even after 100 continuous switching cycles. It indicates the good endurance properties and nonvolatile memory applications for these $\mathrm{Al} / \mathrm{SmO}_{X} / \mathrm{ITO}$ RRAM devices.

\section{Conclusions}

In conclusion, the resistive switching electrical properties of thin-film $\mathrm{Al} / \mathrm{SmO}_{X} / \mathrm{ITO}$ RRAMs were investigated and demonstrated. In addition, as the RF power is $75 \mathrm{~W}$ and the oxygen concentration is $10 \mathrm{sccm}$, the RRAM devices exhibited stable bipolar behavior of greater than $10^{2}$ on/off current ratio, good memory window, and good memory reliability of 100 switching cycles. As the operating voltage is low, the conduction mechanisms for low- and high-resistance states are dominated by ohmic conduction behavior for both SET and RESET states. However, as a higher operating voltage is applied, parts of conduction mechanisms will change to SCLC behaviors. The high uniformity and low-power operation in the $\mathrm{Al} / \mathrm{SmO}_{X} / \mathrm{ITO}$ RRAMs are promising for high-density and low-power electronic applications.

\section{Acknowledgments}

This work was kindly supported by the Ministry of Science and Technology of the Republic of China (MOST 106-2221-E-218-034 and MOST 105-2221-E-218-034).

\section{References}

1 G. I. Meijer: Science 319 (2008) 1625.

2 K. M. Kim, D. S. Jeong, and C. S. Hwang: Nanotechnology 22 (2011) 254002.

3 C. H. Ho, C. L. Hsu, C. C. Chen, J. T. Liu, C. S. Wu, C. C. Huang, C. Hu, and F. L. Yang: Tech. Dig. Int. Electron Devices Meet. (2010) 19.1.1. 
4 R. Waser and M. Aono: Nat. Mater. 6 (2007) 833.

5 S. Z. Rahaman and S. Maikap: Microelectron. Reliab. 50 (2010) 643.

6 S. J. Choi, J. H. Lee, H. J. Bae, W. Y. Yang, T. W. Kim, and K. H. Kim: IEEE Electron Device Lett. 30 (2009) 120.

7 L. Goux, K. Opsomer, R. Degraeve, R. Müller, C. Detavernier, D. J. Wouters, M. Jurczak, L. Altimime, and J. A. Kittl: Appl. Phys. Lett. 99 (2011) 053502.

8 Y. Bernard, P. Gonon, and V. Jousseaume: Appl. Phys. Lett. 96 (2010) 193502.

9 Y. Tsuji, T. Sakamoto, N. Banno, H. Hada, and M. Aono: Appl. Phys. Lett. 96 (2010) 023504.

10 K. Tsunoda, Y. Fukuzumi, J. R. Jameson, Z. Wang, P. B. Griffin, and Y. Nishi: Appl. Phys. Lett. 90 (2007) 113501.

11 H. Choi, M. Pyun, T. W. Kim, M. Hasan, R. Dong, J. Lee, J. B. Park, J. Yoon, D. J. Seong, T. Lee, and H. Hwang: IEEE Electron Device Lett. 30 (2009) 302.

12 S. H. Jo, K. H. Kim, and W. Lu: Nano Lett. 9 (2009) 870.

13 A. K. Kulkarnia, K. H. Schulzb, T. S. Lima, and M. Khanb: Thin Solid Films 308-309 (1997) 1.

14 F. E. Akkad, M. Marafi, A. Punnoose, and G. Prabu: Phys. Status Solidi A 77 (2000) 445.

15 S. K. Choi and J. I. Lee: J. Vac. Sci. Technol. A 19 (2001) 2043.

16 M. Fujimoto, H. Koyama, M. Konagai, Y. Hosoi, K. Ishihara, S. Ohnishi, and N. Awaya: Appl. Phys. Lett. 89 (2006) 223509.

17 S. Seo, M. J. Lee, D. H. Seo, E. J. Jeoung, D. S. Suh, Y. S. Joung, I. K. Yoo, I. R. Hwang, S. H. Kim, I. S. Byun, J. S. Kim, J. S. Choi, and B. H. Park: Appl. Phys. Lett. 85 (2004) 5655.

18 D. C. Kim, M. J. Lee, S. E. Ahn, S. Seo, J. C. Park, I. K. Yoo, I. G. Baek, H. J. Kim, E. K. Yim, J. E. Lee, S. O. Park, H. S. Kim, U. I. Chung, J. T. Moon, and B. I. Ryu: Appl. Phys. Lett. 88 (2006) 232106.

19 T. Oka and N. Nagaosa: Phys. Rev. Lett. 95 (2005) 266403.

20 B. J. Choi, D. S. Jeong, S. K. Kim, C. Rohde, S. Choi, J. H. Oh, H. J. Kim, C. S. Hwang, K. Szot, R. Waser, B. Reichenberg, and S. Tiedke: J. Appl. Phys. 98 (2005) 033715.

21 S. H. Jeon, B. H. Park, J. Lee, B. Lee, and S. Han: Appl. Phys. Lett. 89 (2006) 042904.

22 M. J. Rozenberg, I. H. Inoue, and M. J. Sanchez: Appl. Phys. Lett. 88 (2006) 033510.

23 S. M. Sze: Physics of Semiconductor Devices (Wiley, New York, 1981) 2nd ed., p. 403. 